\title{
Circuit Interpretation and Perturbative Analysis of Differential-to-Common Mode Conversion due to Bend Discontinuities
}

\author{
Xinglong Wu, Flavia Grassi, Sergio A. Pignari \\ Dept. of Electronics, Information and Bioengineering \\ Politecnico di Milano \\ Milan, Italy \\ \{xinglong.wu; flavia.grassi; sergio.pignari\}@polimi.it
}

\author{
Paolo Manfredi, Dries Vande Ginste \\ Dept. of Information technology, IDLab \\ Ghent University-imec \\ Gent, Belgium \\ \{paolo.manfredi; dries.vandeginste\}@ugent.be
}

\begin{abstract}
In this work, the mechanism of differential-tocommon-mode (CM) conversion arising in differential interconnects due to bend discontinuities is investigated. A circuit interpretation of the phenomenon is provided in terms of lumped sources of interference included into the equivalent $\mathrm{CM}$ circuit. The obtained circuit model, assessed through full-wave simulation, allows inferring analogies and differences with respect to other sources of $\mathbf{C M}$ generation such as asymmetries and non-uniformities of the line cross-section, possibly occurring due to the manufacturing process. It is shown that the bent section can be interpreted as a local perturbation of an ideally uniform and symmetric microstrip cross-section and, as such, included into a more general simulation framework based on a perturbative approach aimed at the $\mathrm{CM}$ prediction in differential interconnects.
\end{abstract}

Keywords-Bend discontinuities, common and differential mode, differential interconnects, mode conversion.

\section{INTRODUCTION}

In differential interconnects, the generation of common mode (CM) currents-with consequent degradation of signal integrity and electromagnetic compatibility performancepossibly occurs owing to the imbalance of the two signal lines with respect to ground. Such an imbalance may arise due to tolerances in the value of the involved circuit components, variability in the manufacturing process, as well as design constraints, and has the effect to convert the differential mode (DM) signal into undesired CM (and vice versa) [1]-[3]. In previous works, the DM-to-CM conversion due to an imbalance affecting the line terminations [4] and cross-section (i.e., trace asymmetry [5] and nonuniformity [6]) of differential interconnects was investigated by leveraging the assumption of weak imbalance. By means of such an assumption, it was possible to decouple the solution of the underlying multiconductor transmission line (TL) equations in the modal domain, and to provide the phenomenon of mode conversion with a circuit interpretation in terms of lumped/distributed sources of interference. As a result, a computationally-efficient perturbative approach was developed, allowing fast and accurate prediction of the DM-to-CM conversion due to asymmetry and nonuniformity in differential interconnects [6].

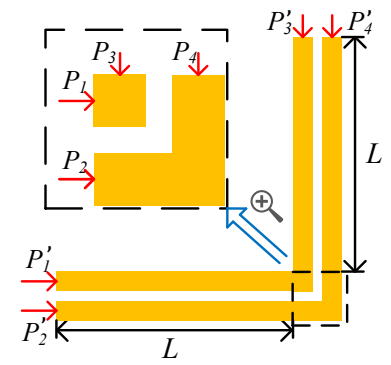

(a)

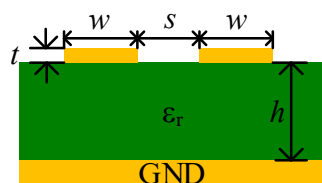

(b)
Fig. 1. Microstrip structure under analysis: (a) Top view and (b) crosssectional view. Geometrical and electrical data: $w=1.75 \mathrm{~mm}, s=0.75 \mathrm{~mm}, t=$ $0.1 \mathrm{~mm}, h=1.5 \mathrm{~mm}, \varepsilon_{r}=4.3, L=25 \mathrm{~mm}$.

In this work, the aforesaid modeling approach is extended to the analysis of the DM-to-CM conversion due to the presence of bend discontinuities. To this end, a differential microstrip with a 90-degree bend is considered. The bend is modeled by a lumped-circuit network [1] with parameters extracted from full-wave simulations. The model is then converted into the modal domain, and simplified by neglecting the back interaction of the $\mathrm{CM}$ onto the DM. This allows modeling the DM-to-CM conversion through lumped voltage and current sources controlled by DM quantities, in analogy with the CM generation due to cross-sectional asymmetries. By this analogy, it is proven that the difference in trace length characterizing the bent section can be modelled as a local nonuniformity affecting an ideal interconnect (which becomes locally unbalanced with respect to ground), and the CM generated by such a discontinuity can be easily predicted by the perturbative algorithms in [6].

\section{Modeling OF BEND DiscontinUities}

To investigate the $\mathrm{CM}$ generation due to bend discontinuities, the differential microstrip shown in Fig. 1 is considered, and assumed to be terminated by perfectly balanced terminal networks, and driven by an ideal DM source $V_{S}$ (Fig. 2). According to [1], the modeling of such a structure is achieved by the cascade connection of the three four-port networks in Fig. 2. Namely, distributed-parameter networks are used to model the two straight portions as perfectly-balanced multi-conductor TL sections with length $L=25 \mathrm{~mm}$, and p.u.l. 


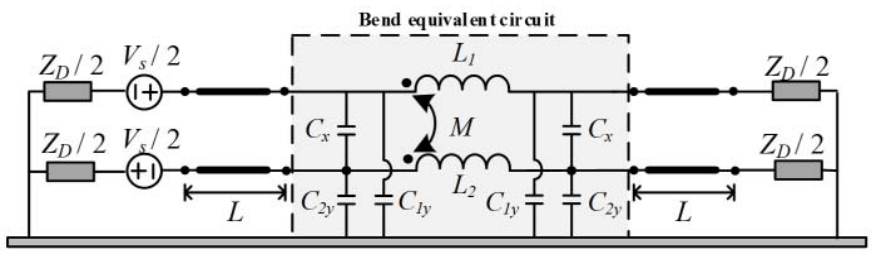

Fig. 2. Equivalent circuit of the bent differential microstrip under analysis.

parameters $l=0.367 \mu \mathrm{H}, l_{m}=0.110 \mu \mathrm{H}, c=96.014 \mathrm{pF}, c_{m}$ $=18.868 \mathrm{pF}$. Conversely, a lumped-parameter circuit is used to provide an approximate representation of the bent section, which holds as long as the bend is electrically short in the frequency range of interest.

To estimate the values of the equivalent circuit, the bent section is implemented in the Keysight Advanced Design System (ADS) Momentum simulator, and characterized by simulation at the four ports $\mathrm{P}_{1}, \mathrm{P}_{2}, \mathrm{P}_{3}, \mathrm{P}_{4}$ [see upper left corner of Fig. 1 (a)]. The obtained $S$-parameters are then used as reference quantities to fit the values of the unknown circuit elements. To this end, the "Swept Optimization" tool available in the ADS Circuit Model Simulation environment is exploited, and the optimization is run in the frequency interval from 10 $\mathrm{MHz}$ up to $3 \mathrm{GHz}$. The obtained values, i.e., $L_{1}=0.072 \mathrm{nH}, L_{2}$ $=1.813 \mathrm{nH}, M=0.069 \mathrm{nH}, C_{1 y}=0.038 \mathrm{pF}, C_{2 y}=0.271 \mathrm{pF}, C_{x}$ $=0.036 \mathrm{pF}$ (subscript 1 and 2 being associated with the shorter and longer trace, respectively), are in line with those obtained in [1], where the model parameters were evaluated by simulating the original microstrip structure, and by deembedding the effects due to the two straight sections from the obtained $S$-parameters. The accuracy of the obtained model in predicting the overall mixed-mode $S$-parameters at the ports of the differential microstrip line under analysis is assessed in Fig. 3. The black curves were obtained by ADS simulation at ports $\mathrm{P}_{1}, \mathrm{P}_{2}, \mathrm{P}_{3}$, and $\mathrm{P}_{4}$. The red curves were predicted by the circuit model of Fig. 2.

\section{CIRCUIT INTERPRETATION OF DM-TO-CM CONVERSION}

To recast the equivalent circuit of Fig. 2 in the modal domain, $\mathrm{CM}$ and $\mathrm{DM}$ voltages and currents are introduced according to the transformation matrices in [7]. This yields uncoupled modal circuits for the terminations as well as for the straight microstrip sections (modelled as two-wire TLs). Conversely, for the bent section, mutually-coupled modal circuits are obtained. As a matter of fact, with reference to Fig. 4(a), the relationship between modal currents (modal voltages are unchanged) at the input and output of the capacitive section reads:

$$
\left(\begin{array}{l}
I_{D M}^{b} \\
I_{C M}^{b}
\end{array}\right)=\left(\begin{array}{l}
I_{D M}^{a} \\
I_{C M}^{a}
\end{array}\right)-j \omega\left[\begin{array}{cc}
C_{D M} & \Delta C \\
\Delta C & C_{C M}
\end{array}\right] \cdot\left(\begin{array}{c}
V_{D M}^{a} \\
V_{C M}^{a}
\end{array}\right),
$$

where $C_{D M}=C_{x}+\left(C_{1 y}+C_{2 y}\right) / 4$ and $C_{C M}=C_{1 y}+C_{2 y}$ are the DM and $\mathrm{CM}$ equivalent capacitances, respectively, whereas the coefficient $\Delta C=\left(C_{l y}-C_{2 y}\right) / 2$ accounts for the undesired conversion from $\mathrm{DM}$ to $\mathrm{CM}$, and vice versa, owing to the difference in length between the two traces.

In a similar fashion, for the pair of coupled inductors in Fig. 4(b), modal voltages are cast as:

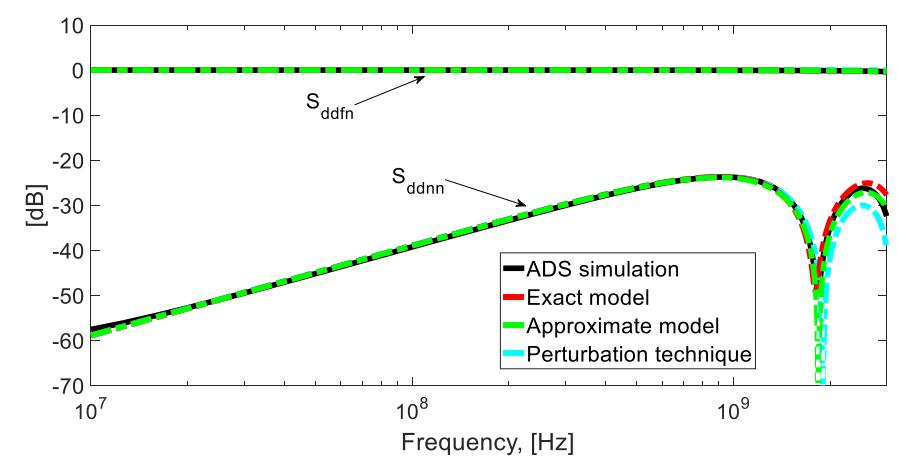

(a)

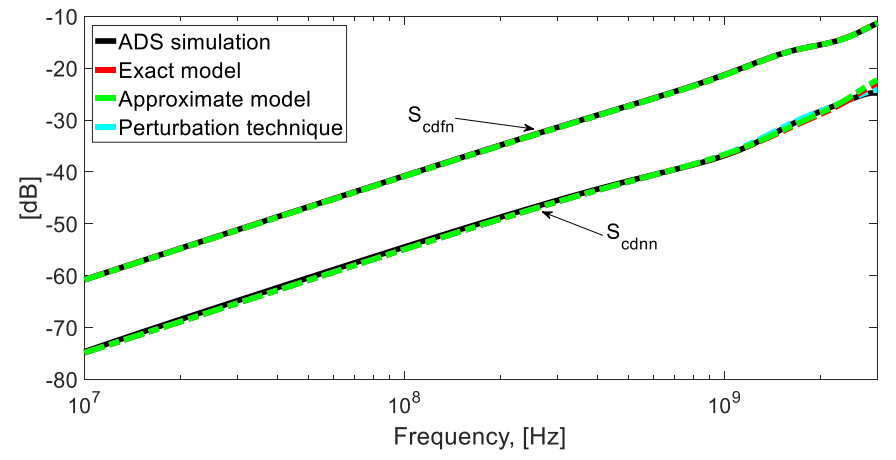

(b)

Fig. 3. Mixed-mode $S$-parameters at the output ports of the bent microstrip DL of Fig. 1. For the simulation: $Z_{D}=100 \Omega$.

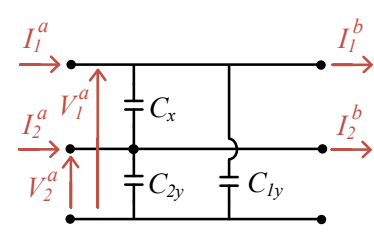

(a)

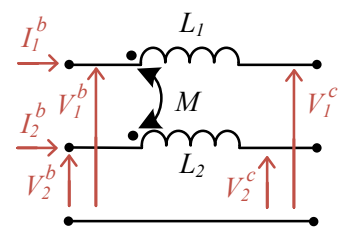

(b)
Fig. 4. Derivation of the equivalent modal circuits associated with the bent section: (a) capacitive section; (b) inductive section.

$$
\left(\begin{array}{l}
V_{D M}^{c} \\
V_{C M}^{c}
\end{array}\right)=\left(\begin{array}{l}
V_{D M}^{b} \\
V_{C M}^{b}
\end{array}\right)-j \omega\left[\begin{array}{cc}
L_{D M} & \Delta L \\
\Delta L & L_{C M}
\end{array}\right] \cdot\left(\begin{array}{l}
I_{D M}^{b} \\
I_{C M}^{b}
\end{array}\right),
$$

where $L_{D M}=L_{1}+L_{2}-2 M$ and $L_{C M}=\left(L_{1}+L_{2}+2 M\right) / 4$ are the $\mathrm{DM}$ and $\mathrm{CM}$ equivalent inductances, respectively, whereas the coefficient $\Delta L=\left(L_{1}-L_{2}\right) / 2$ represents the inductive counterpart of coefficient $\Delta C$ in (1), accounting for mode conversion.

As shown in the following, as long as the bent section is electrically short and even if the assumption of weakimbalance as formulated in [5] is not locally satisfied, the presence of a dominant DM excitation allows achieving an accurate prediction of the $\mathrm{CM}$ by neglecting the $\mathrm{CM}$ backconversion into DM. Hence, the DM and CM circuits can be uncoupled as shown in Fig. 5, and sequentially solved.

The DM circuit of Fig. 5(a) is solved as the first step. Then, the DM voltages and currents in the circuit elements $C_{D M}, L_{D M}$, associated with the bend discontinuity, are evaluated [see quantities marked in red color in Fig. 5(a)]. These quantities are then used as input data for the solution of the CM circuit of Fig. 5(b), where the DM-to-CM conversion is modeled by lumped current and voltage sources 


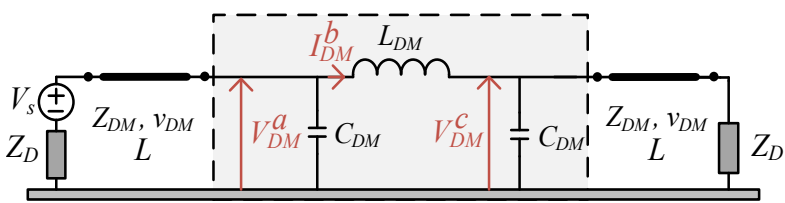

(a)

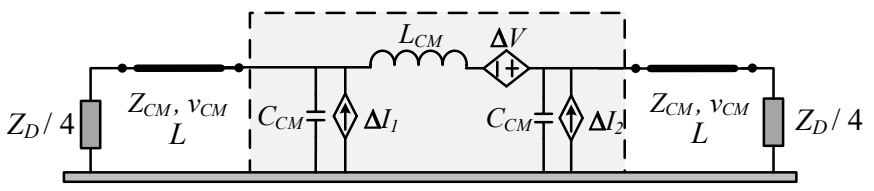

(b)

Fig. 5. Modal circuits for the differential microstrip of Fig. 2: (a) DM and (b) CM. (For the microstrip of Fig. 1: $Z_{D M}=94.475 \Omega, v_{D M}=1.843 \times 10^{8} \mathrm{~m} / \mathrm{s}$, $\left.Z_{C M}=39.314 \Omega, v_{C M}=1.648 \times 10^{8} \mathrm{~m} / \mathrm{s}\right)$

$$
\begin{gathered}
\Delta I_{1,2}=-j \omega \Delta C V_{D M}^{a, c}, \\
\Delta V=-j \omega \Delta L I_{D M}^{b},
\end{gathered}
$$

proportional to the previously-evaluated DM currents and voltages through the imbalance coefficients $\Delta L, \Delta C$.

The accuracy of the approximate modal circuits of Fig. 5 is shown in Fig. 3, where the mixed-mode $S$-parameters predicted by the proposed solution approach (green curves) are compared against ADS full-wave simulations (black curves) and the predictions obtained by exact solution of the circuit of Fig. 2 (red curves) in the interval from $10 \mathrm{MHz}$ up to $3 \mathrm{GHz}$.

\section{ANALOGY WITH LiNE IMBALANCE AND CONCLUSIONS}

The circuit interpretation of DM-into-CM conversion in Fig. 5(b) puts in evidence the strict analogy with the mechanism of $\mathrm{CM}$ generation in microstrip structures with cross-section affected by uniform/nonuniform asymmetries [5], where the phenomenon (and therefore the associated induced sources) is distributed along the line length instead of being lumped in a specific line section.

As a matter of fact, the inner physical network in Fig. 2, as well as the corresponding equivalent $\mathrm{CM}$ and DM circuits in Fig. 5, can be seen as lumped- $\Pi$ representations of an electrically-short equivalent microstrip structure with traces exhibiting cross-sectional imbalance with respect to ground, rather than a different length (as is in the bent section). The strict analogy is further stressed by the expressions of the induced sources in (3), (4), which are formally equivalent to the low-frequency approximation of the induced sources responsible for mode conversion due to line asymmetries, see (29), (30) in [5]. Indeed, the imbalance coefficients $\Delta C, \Delta L$ can be written as $\Delta L=\Delta \ell \cdot L_{a v}, \Delta C=\Delta c \cdot L_{a v} / 2$, where $\Delta \ell, \Delta c$ are the p.u.l. imbalance coefficients of an equivalent microstrip line with length $L_{a v}$ (average length of the bent section, here $L_{a v}=2 w+s$ ) and unbalanced cross-section (i.e., traces with different width).

This analogy allows interpreting bend discontinuities as a local non-uniformity affecting a section (of average length $L_{a v}$ ) of an ideally symmetric (i.e., balanced and straight) differential microstrip line. Hence, the analogy is here leveraged to predict the CM generation in bent differential microstrips by means of the perturbative approach and related algorithms presented in [6]. This yields the mixed-mode $S$-parameters plotted in cyan in Fig. 3, which reveal an appreciable agreement with those predicted by the ADS simulation and by the circuit models of Fig. 2 (red curves) and Fig. 5 (green curves), respectively.

In spite of such an analogy, a significant difference between the two structures is worth to be mentioned here, stemming from the signs of the imbalance coefficients responsible for mode conversion. In fact, in a microstrip with cross-section affected by asymmetries, the imbalance coefficients $\Delta C, \Delta L$ are always opposite in sign. This property can be inferred, in spite of the presence of the dielectric slab, from the p.u.l. inductance $\boldsymbol{L}$ and capacitance $\mathbf{C}$ matrices in free space, whose matrix-product shall satisfy the relationship $\boldsymbol{L} \boldsymbol{C}=c_{0}^{-2} \boldsymbol{I}_{2}, c_{0}$ being the light speed in free space, and $\boldsymbol{1}_{2}$ the $2 \times 2$ identity matrix. Conversely, for bend discontinuities, the two coefficients always exhibit the same sign, since both the selfinductance and capacitance of the longer trace are larger than those of the shorter one.

This reflects in a different combination of the induced voltage and current sources at the two line ends. Namely, in the analogy with crosstalk coupling [5], in asymmetric microstrips the induced voltage and current sources add at the termination closer to the signal source (near end), and subtract at the other termination (far end). Hence, the CM is generally larger at the near end than at the far end. The behavior is opposite in the case of bend discontinuities, as can be appreciated by comparing the mixed-mode $S$-parameters $S_{c d n n}$ and $S_{c d f n}$ in Fig. $3(b)$, which are associated with the near-end and far-end termination, respectively.

\section{REFERENCES}

[1] G.-H. Shiue, W.-D. Guo, C.-M. Lin, R.-B. Wu, "Noise reduction using compensation capacitance for bend discontinuities of differential transmission lines," IEEE Trans. Adv. Packag, vol. 29, no. 3, pp. 560569, Aug. 2006.

[2] C. Gazda, D. Vande Ginste, H. Rogier, R.-B. Wu, D. De Zutter, "A wideband common-mode suppression filter for bend discontinuities in differential signaling using tightly coupled microstrips," IEEE Trans. Adv. Packag, vol. 33, no. 4, pp. 969-978, Nov. 2010.

[3] F. Xiao, K. Murano, and Y. Kami, "Prediction of the electromagnetic radiation from coupled differential microstrip pairs due to skew and imbalance," in Proc. Asia-Pacific Int. Symp. Electromagn. Compat. 2016, Shenzhen, China, May 2016, pp. 683-686.

[4] F. Grassi, G. Spadacini, S. A. Pignari, "The concept of weak imbalance and its role in the emissions and immunity of differential lines," IEEE Trans. Electromagn. Compat., vol. 55, no. 6, pp. 1346-1349, Dec. 2013.

[5] F. Grassi, Y. Yang, X. Wu, G. Spadacini, S. A. Pignari, "On mode conversion in geometrically unbalanced differential lines and its analogy with crosstalk," IEEE Trans. Electromagn. Compat., vol. 57, no. 2, pp. 283-291, Apr. 2015.

[6] X. Wu, F. Grassi, P. Manfredi, D. Vande Ginste, "Perturbative analysis of differential-to-common mode conversion in asymmetric nonuniform interconnects," IEEE Trans. Electromagn. Compat., early access article.

[7] V. K. Tripathi, "Asymmetric coupled transmission lines in an inhomogeneous medium," IEEE Trans. Microw. Theory Tech., vol. 23, no. 9, pp. 734-739, Sep. 1975. 